\title{
Lonoctocog Alfa: A Review in Haemophilia A
}

\author{
Zaina T. Al-Salama ${ }^{1} \cdot$ Lesley J. Scott $^{1}$
}

Published online: 12 September 2017

(C) Springer International Publishing AG 2017

\begin{abstract}
Lonoctocog alfa (rVIII-SingleChain; Afstyla ${ }^{\circledR}$ ) is a novel single-chain recombinant factor VIII (FVIII) molecule, with a truncated B-domain and the heavy and light chains covalently linked to form a stable and homogenous drug that binds with high affinity to von Willebrand factor (VWF). Intravenous lonoctocog alfa is approved for the prophylaxis and treatment of bleeding in patients with haemophilia A in several countries worldwide. In two pivotal, multicentre trials, lonoctocog alfa was effective in the treatment of bleeding episodes and as prophylaxis, including for perioperative management in adults, adolescents and children. In terms of haemostatic efficacy in controlling bleeding episodes, overall treatment and investigatorassessed success rates were high across all age groups, with the majority of these bleeds controlled with a single injection of lonoctocog alfa. Low median spontaneous, overall and traumatic annualized bleeding rates were evident with prophylactic lonoctocog alfa regimens in both trials. Lonoctocog alfa was generally well-tolerated, with very low rates of injection-site reactions. No previously treated patient experienced an anaphylactic reaction or developed an
\end{abstract}

The manuscript was reviewed by: $\boldsymbol{G}$. Castaman, Department of Oncology, Centre for Bleeding Disorders and Coagulation, Careggi University Hospital, Florence, Italy; C. C. Hermans, Division of Haematology, Haemostasis and Thrombosis Unit, Saint-Luc University Clinic, Catholic University of Louvain, Brussels, Belgium; R. Klamroth, Department for Internal Medicine, Haemophilia Treatment Centre, Vivantes Hospital in Friedrichshain, Berlin, Germany; M. Morfini, Past President of Italian Association of Haemophilia Centres (AICE), Florence, Italy.

Zaina T. Al-Salama

demail@springer.com

1 Springer, Private Bag 65901, Mairangi Bay, Auckland 0754, New Zealand inhibitor. In conclusion, lonoctocog alfa is an effective and generally well-tolerated alternative to conventional FVIII products for the treatment and prophylaxis of bleeding, including in the surgical setting, in adults, adolescents and children with haemophilia A.

Lonoctocog Alfa: clinical considerations in haemophilia A

The first and only single-chain recombinant FVIII molecule in which the heavy and light chains are covalently linked to improve stability and affinity for VWF

Provides effective haemostatic efficacy across all age groups, with the majority of bleeds controlled with one injection

Prophylactic use is associated with low median spontaneous, overall and traumatic annualized bleeding rates in patients of all ages

Generally well tolerated; no previously treated patient developed an inhibitor or experienced an anaphylactic reaction

\section{Introduction}

Haemophilia A is an X-linked congenital bleeding disorder caused by mutations in the gene encoding the coagulation factor VIII (FVIII) and represents $80-85 \%$ of the total haemophilia population [1]. The severe form of the disease 
(FVIII levels $<1 \mathrm{IU} / \mathrm{dL}$ ) is characterized by spontaneous bleeding episodes, particularly into the joints (most frequent site of bleeding, accounting for $70-80 \%$ of bleeds) or muscles, predominantly in the absence of an identifiable haemostatic challenge [1]. Bleeding episodes into the joints or the muscles are considered serious, as their recurrence, especially when inadequately treated, can lead to chronic haemophilic arthropathy, a condition associated with chronic pain and disability that remains a challenging and unmet need in the management of patients with haemophilia A [1, 2]. Other manifestations of FVIII deficiency include excessive bleeding episodes following trauma or surgical procedures [1].

The outcomes of haemophilia (prognosis, life expectancy and quality of life) have been substantially improved with early diagnosis, as well as regular prophylactic factor replacement and acute treatment with recombinant or plasma-derived FVIII products [3]. Challenges associated with the stability of conventional recombinant FVIII (rFVIII) products due to dissociation of the heavy and light chains and the role of its carrier protein von Willebrand factor (VWF) [Sect. 6], along with the serious complication of inhibitor development in $30 \%$ of patients with severe haemophilia A, highlight the need for effective new agents with improved stability and reduced immunogenicity $[4,5]$.

Lonoctocog alfa (rVIII-SingleChain; Afstyla ${ }^{\circledR}$ ) is a novel single-chain rFVIII molecule designed to provide an rFVIII with improved stability compared with other available rFVIII products [5-9]. This narrative review discusses the clinical efficacy and tolerability of intravenous lonoctocog alfa for the prevention and treatment of bleeding in children, adolescents and adults with haemophilia A and summarizes its pharmacological properties.

\section{Pharmacological Properties of Lonoctocog Alfa}

\subsection{Mechanism of Action}

Lonoctocog alfa has a truncated FVIII B-domain that allows for the formation of a covalent linkage between the light and heavy chains of FVIII, resulting in a single-chain rFVIII molecule with improved stability compared with other full-length rFVIII products [6]. In addition, modification of the binding site for VWF in lonoctocog alfa enhances its affinity for VWF approximately threefold over full-length rFVIII [5]. VWF stabilizes FVIII and protects it from premature clearance, and may also mitigate the risk of developing inhibitory anti-FVIII antibodies [5, 10, 11].

Treatment with lonoctocog alfa temporarily restores plasma FVIII levels to those required for effective haemostasis and corrects the bleeding tendencies associated with haemophilia A [12-15]. The activated form of lonoctocog alfa has an identical amino acid sequence to that of endogenous FVIII and plays an identical role in the clotting cascade culminating in fibrin clot formation $[14,16]$.

Lonoctocog alfa demonstrated a threefold higher affinity to plasma-derived VWF in preclinical studies relative to full-length rFVIII (dissociation rate constant $=44$ vs. $139 \mathrm{pM}$ ) [5]. In addition to protecting FVIII from proteolysis, evidence from in vitro studies suggests that VWF also plays a role in reducing the development of inhibitors by limiting the recognition of rFVIII by antigen-presenting cells $[10,17]$. In FVIII knockout mice, intravenous lonoctocog alfa significantly $(p=0.006)$ reduced the activated partial thromboplastin time and was associated with a dose-dependent reduction $(p<0.0001)$ in total blood loss, compared with the vehicle control group $[9,16]$. There were no marked differences between lonoctocog alfa and full-length rFVIII (both $20 \mathrm{IU} / \mathrm{kg}$ ) in terms of their corrective actions on thromboelastographic parameters, activated partial thromboplastin time and thrombin generation $[9,16]$. In a thrombosis model, there were no significant differences evident between lonoctocog alfa at doses of 500 and $1000 \mathrm{IU} / \mathrm{kg}$ and B-domain-deleted rFVIII at doses up to $500 \mathrm{IU} / \mathrm{kg}$ in terms of thrombus formation [9].

\subsection{Manufacturing Process}

Lonoctocog alfa is produced by recombinant DNA technology in Chinese hamster ovarian (CHO) cells [13, 16]. The downstream purification of lonoctocog alfa involves a controlled multi-step process, including two dedicated viral removal steps that complement each other in their mode of action [12, 15, 16]. Chromatography, solvent/detergent treatment and serial nanofiltration steps are involved in the purification process to ensure virus safety [16]. There are no human or animal derived proteins used in the formulation or purification processes, and the levels of impurities are effectively and consistently controlled by the manufacturing process [16].

\subsection{Pharmacokinetics}

Following a single intravenous injection of $50 \mathrm{IU} / \mathrm{kg}$ in patients with severe haemophilia A (FVIII activity $<1 \%$ ), lonoctocog alfa demonstrated age-dependent pharmacokinetic (PK) parameters (Table 1). The clearance of lonoctocog alfa decreased with age, with a corresponding increase in the terminal elimination half-life $\left(t_{1 / 2}\right)$ $[8,12,13,16]$. In children aged $<12$ years, $\mathrm{PK}$ parameters were generally similar between the 0 to $<6$ and $\geq 6$ to $<12$ years age groups [8]. 
Table 1 Pharmacokinetic profile of single intravenous lonoctocog alfa doses of $50 \mathrm{IU} / \mathrm{kg}$ in previously treated patients with severe haemophilia A

\begin{tabular}{|c|c|c|c|c|}
\hline \multirow[t]{2}{*}{ Parameter (units) } & \multirow{2}{*}{$\begin{array}{l}\text { Adults }[12,13] 18 \text { to } \leq 65 \text { years } \\
(n=81)\end{array}$} & \multirow{2}{*}{$\begin{array}{l}\text { Adolescents }[12,13] 12 \text { to }<18 \text { years } \\
(n=10)\end{array}$} & \multicolumn{2}{|l|}{ Children [8] } \\
\hline & & & $\begin{array}{l}6 \text { to }<12 \text { years } \\
(n=19)\end{array}$ & $\begin{array}{l}0 \text { to }<6 \text { years } \\
(n=20)\end{array}$ \\
\hline $\mathrm{AUC}_{\infty}(\mathrm{IU} \cdot \mathrm{h} / \mathrm{dL})$ & 1960 & 1540 & 1170 & 1080 \\
\hline $\mathrm{CL}(\mathrm{mL} / \mathrm{h} / \mathrm{kg})$ & 2.90 & 3.80 & 4.63 & 5.07 \\
\hline $\mathrm{C}_{\max }(\mathrm{IU} / \mathrm{dL})$ & 106.0 & 89.70 & 83.50 & 80.20 \\
\hline $\begin{array}{l}\text { IR (IU/dL per } \\
\text { IU/kg) }\end{array}$ & 2.00 & 1.69 & 1.66 & 1.60 \\
\hline MRT (h) & 20.40 & 20.00 & 12.30 & 12.40 \\
\hline $\mathrm{t}_{1 / 2}(\mathrm{~h})$ & 14.20 & 14.30 & 10.20 & 10.40 \\
\hline $\mathrm{V}_{\mathrm{ss}}(\mathrm{mL} / \mathrm{kg})$ & 55.2 & 68.50 & 67.10 & 71.00 \\
\hline
\end{tabular}

FVIII activity assessed using a chromogenic substrate assay; all values are means

$A U C_{\infty}$ area under the FVIII activity-time curve extrapolated to infinity, $C L$ clearance, $C_{\max }$ maximum concentration, FVIII factor VIII, IR incremental recovery, $M R T$ mean residence time, $t_{1 / 2}$ elimination half-life, Vss volume of distribution at steady state

PK parameters of lonoctocog alfa appeared more favourable than those of octocog alfa at equal doses (i.e. single injection of $50 \mathrm{IU} / \mathrm{kg}$ ) in previously treated adults (aged 18-65 years; $n=27$ ) with severe haemophilia A and no history of inhibitors [18]. The FVIII activity PK profiles favoured lonoctocog alfa over octocog alfa, with significantly higher baseline-uncorrected values of mean area under the FVIII activity-time curve extrapolated to infinity $\left(\mathrm{AUC}_{\infty}\right)$ [2090 vs. $\left.1550 \mathrm{IU} \cdot \mathrm{h} / \mathrm{dL} ; p<0.0001\right]$, median time to maximum observed plasma activity $\left(\mathrm{t}_{\max }\right)[0.68 \mathrm{vs} .0 .58 \mathrm{~h}$; $p=0.0014]$, mean $\mathrm{t}_{1 / 2}(14.5$ vs. $13.3 \mathrm{~h} ; p=0.0013)$ and mean residence time (20.4 vs. $17.1 \mathrm{~h} ; p<0.0001)$ [18]. Significantly lower mean clearance (CL) $[2.64$ vs. $3.68 \mathrm{~mL} /$ $\mathrm{h} / \mathrm{kg} ; p<0.0001]$ and mean volume of distribution at steady state $\left(\mathrm{V}_{\mathrm{ss}}\right)[50.0$ vs. $57.1 \mathrm{~mL} / \mathrm{kg} ; p=0.0005]$ were evident with lonoctocog alfa compared with octocog alfa. Similarly, baseline-corrected values were significantly higher for lonoctocog alfa than octocog alfa for mean $\mathrm{AUC}_{\infty}(2030 \mathrm{vs}$. $1440 \mathrm{IU} \cdot \mathrm{h} / \mathrm{dL} ; p<0.0001)$, median $\mathrm{t}_{\max }(0.68$ vs. $0.58 \mathrm{~h}$; $p=0.0014)$, mean $\mathrm{t}_{1 / 2}(14.0$ vs. $11.6 \mathrm{~h} ; p=0.0004)$ and mean residence time (19.6 vs. $15.5 \mathrm{~h} ; p<0.0001)$ [18]. Mean CL ( 2.69 vs. $3.91 \mathrm{~mL} / \mathrm{h} / \mathrm{kg} ; p<0.0001)$ and mean $\mathrm{V}_{\mathrm{ss}}$ $(49.6$ vs. $55.8 \mathrm{~mL} / \mathrm{kg} ; p=0.0028)$ were significantly lower with lonoctocog alfa than with octocog alfa.

In adolescent and adult patients $(n=30)$, the PK profile of lonoctocog alfa after multiple doses (3-6 months' treatment; repeat PK investigation) [4] was generally consistent with that after single doses and did not differ markedly between patients aged $\geq 12$ to $<18$ years and those aged $\geq 18$ years [18]. Lonoctocog alfa demonstrated improved PK parameters versus octocog alfa without glycopegylation or fusion to antibody fragments [18].

In a two-compartment population PK model based on data from two single-dose (lonoctocog alfa $50 \mathrm{IU} / \mathrm{kg}$ ) PK studies in patients with severe haemophilia A (91 patients aged $12-65$ years and 39 aged $<12$ years), predicted PK parameters of lonoctocog alfa-induced FVIII activity correlated with observed PK clinical data [19]. Results using this model support the administration of lonoctocog alfa in doses of 20 and $50 \mathrm{IU} / \mathrm{kg}$ two or three times weekly when used for prophylaxis (Sect. 5). In this model, body weight was a significant covariate on clearance and central distribution volume, and baseline VWF level a significant covariate on clearance, with higher bodyweight and/or baseline VWF levels associated with lower clearance [19].

\section{Therapeutic Efficacy of Lonoctocog Alfa}

The efficacy of intravenous lonoctocog alfa for prophylaxis, perioperative management and treatment of bleeding episodes in previously treated patients with severe haemophilia A was evaluated in two prospective, noncomparative, multicentre trials: a three-part, phase I/III trial in adults and adolescents (aged 12-65 years) [4] (Sect. 3.1) and a phase III trial in children (aged $<12$ years) [8] (Sect. 3.2). The long-term safety and efficacy of lonoctocog alfa is being investigated in an ongoing (completion date 2023), openlabel, multicentre, extension study of these two trials (NCT02172950) [16]. The perioperative management of severe haemophilia A was also evaluated in adults and adolescents [4]. Eligible male patients had to have been treated with a FVIII product for $\geq 150$ exposure days (EDs) [4] or $>50$ EDs [8] prior to enrolment; adults and adolescents who were receiving a FVIII product at the time of enrolment were also included [4]. Patients were excluded if they had detectable inhibitor titre at screening or if they had a personal or family (first-degree) history of FVIII inhibitors [4, 8].

Patients in the routine prophylaxis group received lonoctocog alfa at a dose of 20-40 IU/kg every second day or $20-50 \mathrm{IU} / \mathrm{kg}$ two to three times a week [4] or $15-$ $50 \mathrm{IU} / \mathrm{kg}$ every second day or two to three times a week 
[8]. The prophylactic regimen could also be determined by the investigator $[4,8]$, taking into account the patient's previous treatment regimen prior to enrolment [4], bleeding phenotype [4], historical FVIII dosing [8] and available PK data [8]. World Federation of Hemophilia (WFH) recommendations guided the dosing to treat breakthrough bleeding episodes in patients receiving routine prophylaxis or bleeding in patients receiving on-demand therapy [4, 8]. Doses [4, 8] and frequencies [8] could be adjusted if necessary, but switching between prophylaxis and on demand therapy was not permitted during these trials $[4,8]$. Efficacy analyses were conducted in patients who received $\geq 1$ dose of lonoctocog alfa as part of on-demand treatment or routine prophylaxis $[4,8]$.

\subsection{Adults and Adolescents Aged $\geq 12$ years}

This trial comprised a phase I PK period (part 1; Sect. 2.3), a phase III efficacy and safety period (part 2) and an efficacy, safety and repeat PK period (part 3; Sect. 2.3); it also included a surgical substudy for patients enrolled in parts 2 and 3 [4]. Of the 173 patients in the efficacy population, 27 and 146 patients received on-demand and prophylaxis regimens. At baseline, the median age was 29.5 years, with 100 and $90 \%$ of patients in the on-demand and prophylaxis arms aged $\geq 18$ to $\leq 65$ years; all 14 patients aged $\geq 12$ to $<18$ years were assigned to the prophylaxis group. Haemophilic arthropathy was reported in 56 and $49 \%$ of patients in the on-demand and prophylaxis groups [4].

The co-primary efficacy endpoints were the treatment success rate (i.e. a rating of "excellent" or "good") and the annualized spontaneous bleeding rate (AsBR) in routine prophylaxis, which was compared with on-demand treatment [4]. Treatment success rates were assessed by the investigator for all treated bleeding events (missing data counted as treatment failures) in terms of haemostatic efficacy in the control of bleeding episodes and during surgery, based on a four-point rating scale [4]. The analysis of key efficacy and safety (Sect. 4.1) endpoints was approached in a hierarchical manner using the following order: safety endpoint of inhibitor development (Sect. 4.1) [the upper limit of the $97.5 \% \mathrm{CI}$ for the risk of inhibitor development had to be $\leq 6.8 \%$ in order for further testing to proceed]; treatment success rate for haemostatic efficacy (the lower limit of the $95 \%$ CI for successfully treated bleeds had to be $\geq 70 \%$ ); and the AsBR [16].

A total of 14,306 EDs were accumulated in 174 patients; 120 and 52 of these patients were treated for $\geq 50$ and $\geq 100$ EDs, respectively [4]. Joints were the most frequent location for spontaneous bleeds that required treatment in both groups (91 and $94 \%$ of episodes in the on-demand and prophylaxis groups) [4].

\subsubsection{Control of Bleeding Episodes}

Lonoctocog alfa effectively controlled bleeding episodes, with high treatment success rates for all bleeding episodes (co-primary endpoint) and investigator-assessed bleeding episodes (sensitivity analysis; missing data excluded) and in the on-demand and prophylaxis groups (Table 2) [4]. For the 848 treated bleeding episodes (primary analysis), the haemostatic efficacy of lonoctocog alfa was rated as excellent or good in 71.1 and $21.2 \%$ of episodes; there were no incidences where bleeding episodes did not respond to lonoctocog alfa treatment [16]. Results of sensitivity analyses were generally consistent with those of the primary analysis for the overall population, on-demand and prophylaxis groups [16]. Treatment success rates were also generally similar between the two age groups of $\geq 12$ to $<18$ years and $\geq 18$ to $\leq 65$ years [16].

Of the 848 bleeding episodes treated with lonoctocog alfa (Table 2), all treated bleeding episodes were considered minor or moderate [16]. One or two injections of lonoctocog alfa were sufficient to achieve haemostasis in the majority of bleeding episodes; three or more injections of lonoctocog alfa were required to control bleeding in $<7 \%$ of episodes (Table 2) [4]. Of note, there were no marked differences between the single doses used to achieve haemostatic control and the initial doses used in bleeds requiring multiple injections to achieve haemostatic control [4].

\subsubsection{As Prophylaxis}

For prophylaxis, 54, 32, 6 and $8 \%$ of patients were assigned to three times weekly (median dose $30 \mathrm{IU} / \mathrm{kg}$ ), twice weekly (median dose $35 \mathrm{IU} / \mathrm{kg}$ ), every other day and other regimens of lonoctocog alfa, respectively [4]; the majority (87.7\%) of patients had no or one dose adjustment [16]. The median consumption of lonoctocog alfa across all prophylaxis regimens was $4283 \mathrm{IU} / \mathrm{kg} /$ year [4]. Given that the endpoint of haemostatic efficacy for the treatment of bleeding episodes was met (hierarchical approach), testing for AsBR was carried out.

The prophylactic use of lonoctocog alfa was associated with a median AsBR value of 0.0 [4], which was significantly lower than in patients in the on-demand treatment group (Table 3) [16]. The annualized bleeding rates (ABRs) for overall and traumatic bleeding episodes were also low (with respective median ABRs of 1.14 and 0 ) in the prophylaxis group (Table 3) [4], with $43 \%$ of patients having no bleeding episodes. The median AsBR and ABR did not markedly differ across prophylaxis regimens (i.e. two or three times per week) [4] or age subgroups (i.e. $\geq 12$ to $<18$ or $\geq 18$ to $\leq 65$ years) [16]. The median AsBR 
Table 2 Efficacy of lonoctocog alfa in the treatment of bleeding episodes in the two pivotal multicentre trials in patients with severe haemophilia A

\begin{tabular}{|c|c|c|c|c|c|c|}
\hline \multirow[b]{2}{*}{ Pt population } & \multicolumn{3}{|c|}{ In adults and adolescents aged $\geq 12$ years $[4,12,16]$} & \multicolumn{3}{|c|}{ In children aged 0 to $<12$ years $[8,16]$} \\
\hline & All pts & On-demand & Prophylaxis & All pts & On-demand & Prophylaxis \\
\hline No. of evaluable pts & 173 & 27 & 146 & 83 & 3 & $80^{\mathrm{a}}$ \\
\hline No. of treated bleeding eps (IA/all) & $835 / 848$ & $577 / 590$ & $258 / 258$ & $347 / 347$ & $132 / 132$ & $215 / 215$ \\
\hline \multicolumn{7}{|l|}{ Haemostatic efficacy rating ( $\%$ bleeds) } \\
\hline Excellent or good (all bleeding eps) ${ }^{\mathrm{b}, \mathrm{c}}$ & 92.3 & 92.4 & 92.2 & 96.3 & $100.0^{\mathrm{d}}$ & 94.0 \\
\hline Moderate (all bleeding eps) ${ }^{\mathrm{e}}$ & 6.1 & 5.4 & 7.8 & 3.5 & 0 & 5.6 \\
\hline Excellent or good (IA bleeding eps) ${ }^{\mathrm{c}}$ & 93.8 & 94.5 & 92.2 & 96.3 & $100.0^{\mathrm{d}}$ & 94.0 \\
\hline Moderate (IA bleeding eps) ${ }^{\mathrm{e}}$ & 6.2 & 3.8 & 7.8 & 3.5 & 0 & 5.6 \\
\hline \multicolumn{7}{|l|}{ Bleeding eps $(\%)$ controlled with ${ }^{f}$} \\
\hline $1 \mathrm{inj}$ & 80.9 & 82.7 & 76.7 & 85.9 & 99.2 & 77.7 \\
\hline 2 injs & 12.6 & 12.0 & 14.0 & 9.8 & 0 & 15.8 \\
\hline$\geq 3$ injs & 6.5 & 5.3 & 9.3 & 4.3 & 0.8 & 6.5 \\
\hline Overall med dose/inj/bleeding ep (IU/kg) & 31.7 & 30.0 & 31.0 & 27.3 & 25.9 & 30.0 \\
\hline Overall med cumul dose/bleeding ep (IU/kg) & 34.7 & - & - & 27.6 & 25.9 & $37.0^{\mathrm{g}}$ \\
\hline
\end{tabular}

Cumul cumulative, $e p(s)$ episode(s), IA investigator-assessed, $\operatorname{inj}(s)$ injection(s), LON lonoctocog alfa, med median, $p t(s)$ patient(s)

${ }^{\text {a }}$ Prophylaxis regimens were: every second day $(n=3)$, three times weekly $(n=24)$, twice weekly $(n=43)$, and other regimens $(n=10)$

${ }^{\mathrm{b}}$ Primary endpoint; missing data counted as treatment failures

${ }^{\mathrm{c}}$ Excellent $=$ definite pain relief \pm improvement in signs of bleeding within $\approx 8 \mathrm{~h}$ post 1 st inj of LON; good $=$ definite pain relief \pm improvement in signs of bleeding at $\approx 8 \mathrm{~h}$ post $1 \mathrm{st}$ inj, but required 2 injs for complete resolution

${ }^{\mathrm{d}}$ Haemostatic efficacy was assessed as excellent in all (100\%) treatment procedures

e Probable/slight beneficial effect within $\approx 8 \mathrm{~h}$ post 1 st inj of LON, requires $>2$ injs for complete resolution

${ }^{\mathrm{f}}$ Secondary endpoint. Based on all treated bleeding episodes; missing ratings counted as treatment failures

g Across all prophylaxis regimens, the median prophylaxis dose/subject was $342 \mathrm{IU} / \mathrm{kg} / \mathrm{month}$ and $4109 \mathrm{IU} / \mathrm{kg} / \mathrm{year}$

was 0.0 in patients receiving two or three times weekly prophylaxis, with respective median observed ABRs of 0.0 and 1.93 [4]. The AsBR and ABR for adolescents (aged $\geq 12$ to $<18$ years) receiving prophylaxis versus on- demand regimens could not be compared, as all adolescents received the prophylaxis regimen [16]. Table 3 summarizes the ABRs (spontaneous, overall, and for traumatic bleeds) in the on-demand group.

Table 3 Efficacy of lonoctocog alfa in the prevention or reduction of bleeding episodes in patients with severe haemophilia A: results from the two pivotal trials

\begin{tabular}{|c|c|c|c|c|}
\hline \multirow[b]{2}{*}{ Pt population } & \multicolumn{2}{|c|}{$\begin{array}{l}\text { In adults and adolescents aged } \geq 12 \text { years } \\
{[4,12,16]}\end{array}$} & \multicolumn{2}{|c|}{$\begin{array}{l}\text { In children aged } 0 \text { to }<12 \text { years } \\
{[8,12,16]}\end{array}$} \\
\hline & On-demand & Prophylaxis & On-demand ${ }^{\mathrm{a}}$ & Prophylaxis \\
\hline No. of evaluable pts & 27 & 146 & 3 & 80 \\
\hline Pts $(\%)$ with no treated bleeding episodes & 3.7 & 43.2 & 0 & 26.3 \\
\hline Med AsBR ${ }^{b}$ & $11.73^{\mathrm{c}}$ & $0.00 * \mathrm{c}$ & 31.76 & 0 \\
\hline Med (overall) ABR & $19.64^{\mathrm{d}}$ & $1.14 * \mathrm{~d}$ & $78.56^{\mathrm{d}}$ & $3.69^{\mathrm{d}}$ \\
\hline Med ABR for traumatic bleeds & $3.12^{\mathrm{d}}$ & $0^{\mathrm{d}}$ & 35.12 & 1.97 \\
\hline
\end{tabular}

$A B R$ annualized bleeding rate, $A s B R$ annualized spontaneous bleeding rate, med median, $p t(s)$ patient(s)

$* p<0.0001$ vs. pts in the on-demand treatment group

a All on-demand recipients were aged $\geq 6$ to $<12$ years; no formal comparisons of ABR between groups were performed

b $\mathrm{ABR}[4,8]$ and AsBR [4] = no. of treated events $\times 365.25$ /efficacy evaluation period (excluded data from the pharmacokinetics and the surgical parts [4])

c Co-primary efficacy endpoint

d Secondary endpoint 
An analysis of the 48 patients who had previously received a prophylaxis regimen indicates that 35 and $38 \%$ of patients were receiving two or three times weekly prophylaxis at the end of the study (vs. 12 and 52\% pre-study), while 19 and $8 \%$ of patients were receiving other regimens of prophylaxis therapy or every second day at the end of study (vs. 17 and 19\%) [4].

\subsubsection{Perioperative Prophylaxis}

During the trial, 13 adults and adolescents underwent a total of 16 elective major surgeries (including seven joint surgeries/replacements) that required general, spinal or regional anaesthesia (two of which were performed in one session but received differentiated haemostasis assessment) [4]. Lonoctocog alfa dosing regimens were individualized based on the type of surgery and clinical status of the patient and were adjusted to achieve and maintain FVIII activity levels recommended by the WFH guidelines [4].

Perioperative prophylaxis with lonoctocog alfa maintained effective haemostasis in all patients, with investigator-assessed haemostatic efficacy in surgical prophylaxis rated as excellent in 94\% of surgeries [4]. An excellent rating was defined as haemostasis clinically not significantly different from normal in the absence of other haemostatic intervention, and estimated blood loss not $>20 \%$ higher than the predicted blood loss for the intended surgery. The median lonoctocog alfa consumption during the preoperative and intraoperative periods to maintain haemostasis was $89.4 \mathrm{IU} / \mathrm{kg}$ [4], which was within expectations for the types of procedures performed [16].

\subsection{Children Aged $<12$ years}

Previously treated children $(n=84 ; 35$ patients aged 0 to $<6$ years, 49 patients aged $\geq 6$ to $<12$ years) were assigned by the investigator to lonoctocog alfa prophylaxis $(n=81$; 1 patient was excluded from the efficacy population) or ondemand therapy $(n=3)$ [8]. At baseline, the median age was 7 years; demographic characteristics were generally similar in the two age cohorts; predictably, median age (9 vs. 4 years) and bodyweight ( $32 \mathrm{vs.} 16 \mathrm{~kg}$ ) were approximately twofold higher in the $\geq 6$ to $<12$ years cohort than in the 0 to $<6$ years cohort. The primary endpoint was treatment success, as assessed by the investigator for all treated bleeding episodes [8].

The proportions of children in the $\geq 6$ to $<12$ and 0 to $<6$ years cohorts requiring $\geq 1$ dose adjustment were 44 and $31 \%$; five patients in the $\geq 6$ to $<12$ years and one in the 0 to $<6$ years cohort required $>2$ dose adjustments [16]. Joints were the most frequent $(61.7 \%)$ location for bleeding episodes. A total of 5239 EDs were accumulated; 65 and 8 patients were treated for $\geq 50$ and $\geq 100$ EDs [8].
Initially, the most frequently assigned prophylaxis regimens (across all age groups) were the twice weekly (43 of 80 patients) and three times weekly ( 24 patients) regimens, with 38 and 32 patients assigned to these respective regimens by study end [16]. The majority $(92.5 \%)$ of patients received an initial dose of $15-50 \mathrm{IU} / \mathrm{kg}$, with the remaining patients assigned to doses not exceeding $57 \mathrm{IU} / \mathrm{kg}$ [16].

\subsubsection{Control of Bleeding Episodes}

Lonoctocog alfa provided effective control of bleeding episodes in children, with high treatment success rates for all treated bleeding events (primary endpoint) [all 347 episodes were investigator-assessed] and in the on-demand and prophylaxis groups (Table 2) [8]. In the primary analysis, haemostatic efficacy was rated as excellent and good in 85.3 and $11 \%$ of bleeding episodes [8]. Results of sensitivity analyses of haemostatic efficacy were also generally consistent with those in the primary analysis [16]. One injection of lonoctocog alfa was sufficient to control the majority of bleeding episodes (Table 2) [8]. The treatment success rates were generally similar between patients aged $\geq 6$ to $<12$ years and 0 to $<6$ years ( 97 and $94 \%$ ), as was the proportion of bleeding episodes controlled with one or two injections in these cohorts (96 and 94\%) [16].

\subsubsection{As Prophylaxis}

Lonoctocog alfa prophylaxis was associated with low median ABRs (for overall and traumatic bleeds) and AsBR (Table 3) [8]. The median ABR for joint bleeding episodes was 2.91 [16], with $26 \%$ of patients having no bleeding episodes [12]. The median observed ABR was 2.4-fold higher in the $\geq 6$ to 12 years group than in the 0 to $<6$ years group (5.1 vs. 2.1) and was driven primarily by traumatic bleeds; the respective median observed ABRs for joint bleeding episodes were 2.3 versus $0.0[8,16]$. The median AsBR was 0.0 for both age groups $[8,16]$, with 16 versus $40 \%$ of patients aged $\geq 6$ to $<12$ years and 0 to $<6$ years having no bleeding episodes [16]. The median ABRs (for overall and traumatic bleeds) and AsBR in the on-demand treatment group are shown in Table 3 [8]. In the on-demand treatment group, the median ABR for joint bleeding episodes was 43.51 [16]. There were no patients with zero treated bleeding episodes in the on-demand treatment group.

In patients receiving twice weekly (median initially prescribed dose $35 \mathrm{IU} / \mathrm{kg}$ ) and three times weekly (median prescribed dose $32 \mathrm{IU} / \mathrm{kg}$ ) prophylaxis regimens, the median observed ABRs for total bleeding episodes were 4.4 and 2.3, the median observed AsBR was 0.0 for both 
regimens and the median joint $\mathrm{ABRs}$ were 1.93 and 0.82 [8]. Although doses up to $50 \mathrm{IU} / \mathrm{kg}$ were permitted, the prescribed doses per injection were not higher in the group receiving twice weekly prophylaxis, resulting in a $30 \%$ overall lower consumption in these recipients [16]. No conclusive comparisons were made with other regimens, as the numbers of patients in each of these groups were too small $(n=3-10)$ [16]. There were no treated bleeding episodes in 15.0 and $37.5 \%$ of patients receiving two or three times weekly prophylaxis regimens [16].

With two or three times weekly prophylaxis regimens, 39.5 and $41.7 \%$ of patients had at least one dose adjustment, with respective median doses of 35.5 and $32 \mathrm{IU} / \mathrm{kg}$ at study end [8]. Few patients increased the frequency of their injections from twice $(n=6)$ or once $(n=1)$ weekly to three times weekly; one patient changed their injection frequency from every other day to three times a week [8]. Data available for 48 patients who received prophylaxis prior to study entry indicate that $35 \%$ of these patients reduced their injection frequency compared with their prestudy regimen [8].

In the children who had a positive test for non-inhibitory anti-drug antibodies (ADAs) [i.e. anti-FVIII immunoglobulin (Ig) $\mathrm{G}$ and/or IgM antibodies] at any time during the study $(n=19)$, there were no marked differences in terms of the observed median ABR (2.73) or percentage of patients with zero bleeding episodes (32\%) compared with the overall prophylaxis population [8].

\section{Tolerability of Lonoctocog Alfa}

Lonoctocog alfa was generally well tolerated in clinical trials discussed in Sect. 3 in patients with severe haemophilia A. In these two trials ( $n=258$ in the overall safety population; total EDs 28,418 ; $\geq 28,492$ injections), 185 patients had $\geq 50$ EDs, 60 of whom had $\geq 100$ EDs [12]. In the clinical program (i.e. inclusive of the ongoing extension study), 109 patients had $\geq 100$ EDs [16].

Although $65-76 \%$ of participants in the two pivotal trials experienced treatment-emergent adverse events (TEAEs), the majority of these were rated as unrelated to the study drug and were mild or moderate in severity [4, 8]. The most commonly reported adverse events (AEs) in adults and adolescents [4] or children (no quantitative data reported) [8] were nasopharyngitis (10.3\% [4]), arthralgia (9.8\% [4]), headache (6.9\% [4]) and cough [8]. The incidence of treatment-related adverse events (TRAEs) was low $(7.5 \%$ of patients [4]; $1.2 \%$ [8]). The most commonly reported TRAEs (reported in $>0.5 \%$ of patients) in the overall safety population were hypersensitivity $(1.6 \%$ of patients) and dizziness $(0.8 \%)$; other TRAEs included paresthesia, rash, erythema, pruritus, pyrexia, injection-site pain, chills and feeling hot (all 0.4\%) [12]. Rates of injection-site reactions were very low (0.7 [4], 0.6 [8] and $0.1 \%$ in the extension study [16]), with no reports of severe local reactions in any study.

There were no age-specific differences in adverse reactions observed between adults, adolescents and children [13]. No patients withdrew from the trial in adults and adolescents [4] or long-term extension study [16] due to AEs; one patient withdrew from the trial in children [8] due to hip arthralgia (not treatment-related). AEs were reported by $31 \%$ of patients in the long-term extension trial [16].

Treatment-emergent serious AEs were reported in 4.0 [4] and $10.7 \%$ [8] of patients, with these events considered to be treatment-related in $0.6 \%$ (one case of hypersensitivity) [4] and $0 \%$ [8] of patients. The serious TEAE of hypersensitivity was managed with steroids and antihistamines, allowing same-day discharge [4]. Given that allergic-type hypersensitivity reactions are possible with lonoctocog alfa, patients must be informed to recognize early signs of hypersensitivity, which may progress to anaphylaxis (shock) $[12,13]$. Patients should be advised to discontinue lonoctocog alfa [12, 13] and contact their physician [13] if symptoms of hypersensitivity occur $[12,13]$; pre-medication may be considered in patients with previous hypersensitivity reactions $[12,13]$. In the ongoing, long-term extension study (interim data as of 29 May 2015), serious AEs were reported by $\approx 3 \%$ of patients, although none were considered to be treatment-related and all were resolved [16]. There were no occurrences of anaphylaxis or thrombosis reported in the two pivotal trials [12] and no deaths have occurred [16].

\subsection{Immunogenicity}

The (cumulative [8]) rate of inhibitor formation to FVIII was the primary safety endpoint for the trials in adults and adolescents [4] and in children [8]; inhibitor formation was defined as an antibody titre of $\geq 0.6$ Bethesda units (BU)/ $\mathrm{mL}$ after lonoctocog alfa $[8,16]$. No FVIII inhibitors were detected in any previously treated patients in either trial (including 120 patients with $\geq 50$ EDs [4] and 65 patients with $\geq 50$ EDs [8]). One patient with a pre-existing inhibitor whose inhibitor titre was incorrectly reported as negative at screening entered the study with a low titre inhibitor (3.46 BU/mL); following an intensified prophylaxis regimen of $50 \mathrm{IU} / \mathrm{kg}$ three times weekly, the patient became inhibitor-negative at $\approx 3$ months, which was maintained at the end of the study [8].

No individual had pre-existing anti-CHO antibodies or developed these during either trial $[4,8]$. In terms of the development of ADAs in the trial in adults and adolescents, four patients who were negative at screening developed ADAs during the trial, two of whom were negative at study 
end [4]; during the other study, ten children developed ADAs, three of whom were negative for ADAs at study end [12]. There were no AEs associated with the development of ADAs [12]. In the extension study, no further patients developed an ADA [16].

\section{Dosage and Administration of Lonoctocog Alfa}

Intravenous lonoctocog alfa is approved in several countries worldwide. In the EU, it is approved for the treatment and prophylaxis of bleeding in patients (all age groups) with haemophilia A [13] and, in the USA, for use in adults and children with haemophilia A for on-demand treatment and control of bleeding episodes, routine prophylaxis to reduce the frequency of bleeding episodes and perioperative management of bleeding [12]. The rate of injection of the reconstituted preparation should be slow at a rate comfortable to the patient, not exceeding $10 \mathrm{~mL} / \mathrm{min}$ $[12,13]$. The clinical effectiveness of lonoctocog alfa may vary in individual cases and is an important consideration for the amount and frequency of administered treatment; the dose and duration of treatment depends on the severity of the FVIII deficiency, the location and extent of bleeding and the patient's clinical condition [12, 13]. Consideration should be given to maintaining FVIII activity at or above the target range in patients with certain haemorrhagic events (e.g. life-threatening haemorrhages), including surgery (major and minor surgery such as tooth extraction) $[12,13]$.

In patients receiving (routine [12]) prophylaxis, the lonoctocog alfa dosing regimen may be adjusted based on patient response $[12,13]$. The recommended lonoctocog alfa dosage is $20-50 \mathrm{IU} / \mathrm{kg}$ two or three times weekly in adults and adolescents ( $\geq 12$ years), and $30-50 \mathrm{IU} / \mathrm{kg}$ two to three times weekly in children $(<12$ years); children may require more frequent or higher doses to compensate for higher clearance in this age group (Sect. 2.3) [12, 13]. Local prescribing information should be consulted for information regarding regimen recommendations, reconstitution and administration procedures, use in specific patient populations, contraindications, special warnings and precautions.

\section{Current Status of Lonoctocog Alfa in Haemophilia A}

The cornerstone in the management of haemophilia $\mathrm{A}$ is the prevention and treatment of bleeding episodes, preferably through replacement of the deficient clotting factor (i.e. FVIII) and prompt treatment of acute bleeds $(\leq 2 \mathrm{~h})$ [1]. Although the advent of rFVIII products has been a major advance in haemophilia A management, these products are limited by a lack of stability, which necessitates frequent administration and thereby increases the burden of treatment when they are used for prophylaxis [20]. Accordingly, various technologies are being pursued to improve treatment options for patients with haemophilia A, including single-chain design, $\mathrm{Fc}$ fusion and direct glycopegylation [20]. However, technologies that extend the half-life of FVIII (e.g. Fc fusion and direct glycopegylation) are still limited to an increase of 1.5-1.7 times the normal half-life of endogenous FVIII, mainly due to the dependence of FVIII on the half-life of VWF in the circulation [4]; VWF also protects FVIII from antigen-presenting cells and stimulation of immune effector cells [6]. Therefore, improving the affinity of FVIII for VWF enhances the FVIII-VWF interaction and can improve the therapeutic profile of the agent [6].

Lonoctocog alfa is the first and only single-chain rFVIII molecule in which heavy and light chains are covalently linked (Sect. 2.1) to form a stable and homogenous drug product that binds with high affinity to VWF. This provides favourable PK properties compared with full-length rFVIII (octocog alfa); the single-chain construct also increases stability of the rFVIII and increases its affinity for VWF, thereby stabilizing FVIII and protecting it from premature clearance (Sect. 2.1).

Administered dose and frequency of repeat injections of lonoctocog alfa may be guided by plasma FVIII activity as monitored using the chromogenic or one-stage clotting assay, although the former assay more accurately reflects the clinical haemostatic potential of this agent and is thus the preferred option $[12,13,21]$. Relative to the chromogenic assay, using the one-stage clotting assay underestimates lonoctocog alfa by $\approx 50 \%$ [22]; therefore, the result must be multiplied by a factor of two to determine the patient's FVIII activity level $[12,13]$. It must be noted that the discrepancy observed between the chromogenic and one-stage assays varies between products [22], and that monitoring with one-stage assays is not suitable for some novel modified rFVIII products [23].

In two pivotal trials, lonoctocog alfa was effective in the treatment of bleeding episodes and as prophylaxis, including for perioperative management of bleeds (Sect. 3), in adults and adolescents (Sect. 3.1) and children (Sect. 3.2). In terms of haemostatic efficacy in controlling bleeding episodes, overall treatment and investigator-assessed success rates were high across all age groups ( $\geq 92 \%$ in adults/adolescents and $\geq 94 \%$ in children), with the majority of these bleeds controlled with a single injection of lonoctocog alfa (Table 2). As prophylaxis, lonoctocog alfa was associated with median AsBRs of zero in both pivotal trials and low median ABRs (Sects. 3.1.2 and 3.2.1), including for overall and traumatic ABRs (Table 3). 
In children, there were no marked differences in ABRs or percentages of patients with zero bleeding episodes between patients with positive non-inhibitory ADAs at any time during the trial and the overall prophylaxis population (Sect. 3.2.2). Children aged $\geq 6$ to $<12$ years had a 2.4 -fold higher median ABR than those aged 0 to $<6$ years, with the difference primarily driven by traumatic bleeds (Sect. 3.2.2). The median ABR is low with three times weekly prophylaxis (Sect. 3.2.1), and the 1.9-fold higher ABR observed in patients receiving two times weekly prophylaxis potentially reflects the generally higher clearance in children (Table 1). The $30 \%$ overall lower consumption with twice weekly prophylaxis (Sect. 3.2.1) could have further contributed to the ABR value.

The benefits of improving the stability of lonoctocog alfa and its high binding affinity to VWF translating into meaningful clinical outcomes (i.e. less frequent administration) were supported by results from the two pivotal trials [4, 8]. A comparison of pre- and end-of-study treatment regimens in adults and adolescents treated with prophylaxis therapy prior to enrolment indicated that at the end of the study, fewer patients were receiving every second day or three times weekly regimens, whereas the number of patients receiving twice weekly prophylaxis increased by approximately threefold (Sect. 3.1.2). Similarly, 35\% of children previously treated with prophylaxis prior to study entry had a reduction in their injection frequency compared with their pre-study regimen (Sect. 3.2.2).

Lonoctocog alfa was generally well tolerated in adults, adolescents and children with haemophilia A (Sect. 4). The rates of injection-site reactions were low $(<1 \%)$ and no patient experienced an anaphylactic reaction (Sect. 4) or developed a FVIII inhibitor or antibodies against $\mathrm{CHO}$ host cell proteins (Sect. 4.1). Given the involvement of $\mathrm{CHO}$ cells in the manufacturing process of lonoctocog alfa (Sect. 2.1), the drug is contraindicated in patients with a known allergy to hamster protein $[12,13]$. The EU summary of product characteristics states that the risk of developing inhibitors is related to the exposure to FVIII, particularly within the first 20 EDs [13]. Although the risk of inhibitor development is very low after the first 100 EDs, cases of recurrent inhibitors have been observed when switching between FVIII products in previously treated patients with $>100$ EDs and a history of inhibitor development; therefore, it is recommended that all patients are carefully monitored for inhibitor occurrence following any product switch [13]. In the event that the expected FVIII activity plasma levels are not attained or if bleeding is not controlled with an appropriate dose, testing for the presence of FVIII inhibitors should be performed [12, 13]; consideration must be given to other treatment options in patients with high levels of inhibitor [13]. Given that the risk of inhibitor formation is increased in previously untreated patients with haemophilia compared with previously treated patients [16], results from the ongoing longterm extension trial (NCT02172950) that includes this population would be of interest and relevance to the management of haemophilia A.

Pharmacoeconomic analyses of lonoctocog alfa versus conventional FVIII products would be of interest, as would health-related quality of life assessments.

In conclusion, in the two pivotal trials, intravenous lonoctocog alfa was an effective and generally well tolerated treatment option for preventing and controlling bleeding episodes, including in the surgical setting, in adults, adolescents and children with haemophilia A. Thus, lonoctocog alfa is an effective and well tolerated alternative to conventional FVIII products in the management of haemophilia A.

\section{Data Selection Lonoctocog Alfa: 69 records identi- fied \\ Duplicates removed 8 \\ Excluded at initial screening (e.g. press releases; news reports; not relevant drug/indication) \\ Excluded during initial selection (e.g. preclinical study; reviews; case reports; not randomized trial) \\ Excluded during writing (e.g. reviews; duplicate data; small patient number; nonrandomized/phase I/II trials) \\ Cited efficacy/tolerability articles \\ Search Strategy: EMBASE, MEDLINE and PubMed from 1946 to present. Clinical trial registries/databases and websites were also searched for relevant data. Key words were Lonoctocog, Afstyla, CSL627, rVIII, rFVIII, recombinant, single-chain, hemophilia A, haemophilia A. Records were limited to those in English language. Searches last updated 4 September 2017}

Acknowledgements During the peer review process, the manufacturer of lonoctocog alfa was also offered an opportunity to review this article. Changes resulting from comments received were made on the basis of scientific and editorial merit.

\section{Compliance with Ethical Standards}

Funding The preparation of this review was not supported by any external funding.

Conflict of interest Zaina T. Al-Salama and Lesley J. Scott are salaried employees of Adis/Springer, are responsible for the article content and declare no relevant conflicts of interest.

Additional information about this Adis Drug Review can be found at http://www.medengine.com/Redeem/CBD8F060224F2E65. 


\section{References}

1. Srivastava A, Brewer AK, Mauser-Bunschoten EP, et al. Guidelines for the management of hemophilia. Haemophilia. 2013;19(1):e1-47.

2. Manco-Johnson MJ, Riske B, Kasper CK. Advances in care of children with hemophilia. Semin Thromb Hemost. 2003;29(6):585-94.

3. Srivastava A. Haemophilia care-beyond the treatment guidelines. Haemophilia. 2014;20(Suppl 4):4-10.

4. Mahlangu J, Kuliczkowski K, Karim FA, et al. Efficacy and safety of rVIII-SingleChain: results of a phase 1/3 multicenter clinical trial in severe hemophilia A. Blood. 2016;128(5):630-7.

5. Zollner S, Raquet E, Claar P, et al. Non-clinical pharmacokinetics and pharmacodynamics of rVIII-SingleChain, a novel recombinant single-chain factor VIII. Thromb Res. 2014;134(1):125-31.

6. Schulte S. Pioneering designs for recombinant coagulation factors. Thromb Res. 2011;128(Suppl 1):S9-12.

7. Schulte S. Innovative coagulation factors: albumin fusion technology and recombinant single-chain factor VIII. Thromb Res. 2013;131(Suppl 2):S2-6.

8. Stasyshyn O, Khayat CD, Iosava G, et al. Safety, efficacy and pharmacokinetics of rVIII-SingleChain in children with severe hemophilia A: results of a multicenter clinical trial. J Thromb Haemost. 2017;15(4):636-44.

9. Zollner SB, Raquet E, Müller-Cohrs J, et al. Preclinical efficacy and safety of rVIII-SingleChain (CSL627), a novel recombinant single-chain factor VIII. Thromb Res. 2013;132(2):280-7.

10. Delignat S, Repesse Y, Navarrete AM, et al. Immunoprotective effect of von Willebrand factor towards therapeutic factor VIII in experimental haemophilia A. Haemophilia. 2012;18(2):248-54.

11. Pipe SW, Montgomery RR, Pratt KP, et al. Life in the shadow of a dominant partner: the FVIII-VWF association and its clinical implications for hemophilia A. Blood. 2016;128(16):2007-16.

12. CSL Behring GmbH. Afstyla ${ }^{\circledR}$ [antihemophilic factor (recombinant), single chain for intravenous injection, powder and solvent for injection]: US prescribing information. 2016. http://labeling. cslbehring.com. Accessed 4 Sept 2017.

13. CSL Behring GmbH. Afstyla ${ }^{\circledR}$ (lonoctocog alfa): EU summary of product characteristics. 2016. https://ec.europa.eu/. Accessed 04 Sept 2017.

14. US FDA. BLA Clinical review memorandum. 2015. https://www. fda.gov/. Accessed 4 Sept 2017.

15. US FDA. Clinical pharmacology BLA review. 2015. http://www. fda.gov/. Accessed 4 Sept 2017.

16. European Medicines Agency. Afstyla (lonoctocog alfa): EU assessment report. 2016. http://www.ema.europa.eu/. Accessed 4 Sept 2017.

17. Dasgupta S, Repesse Y, Bayry J, et al. VWF protects FVIII from endocytosis by dendritic cells and subsequent presentation to immune effectors. Blood. 2007;109(2):610-2.

18. Klamroth R, Simpson M, von Depka-Prondzinski M, et al. Comparative pharmacokinetics of rVIII-SingleChain and octocog alfa $\left(\right.$ Advate $\left.^{\circledR}\right)$ in patients with severe haemophilia A. Haemophilia. 2016;22(5):730-8.

19. Zhang Y, Roberts J, Tortorici M, et al. Population pharmacokinetics of recombinant coagulation factor VIII-SingleChain in patients with severe hemophilia A. J Thromb Haemost. 2017;15(6):1106-14.

20. Mannucci PM. Half-life extension technologies for haemostatic agents. Thromb Haemost. 2015;113(1):165-76.

21. Peyvandi F, Oldenburg J, Friedman KD. A critical appraisal of one-stage and chromogenic assays of factor VIII activity. J Thromb Haemost. 2016;14(2):248-61.

22. Veldman A, Feussner A, Kalina U, et al. rVIII-SingleChain plasma activity can be measured using both the one-stage and the chromogenic substrate assay: results from an international field study [abstract plus poster]. In: 61st annual meeting of the society of thrombosis and hemostasis Research. 2017.

23. Kitchen S, Kershaw G, Tiefenbacher S. Recombinant to modified factor VIII and factor IX-chromogenic and one-stage assays issues. Haemophilia. 2016;22(Suppl 5):72-7. 